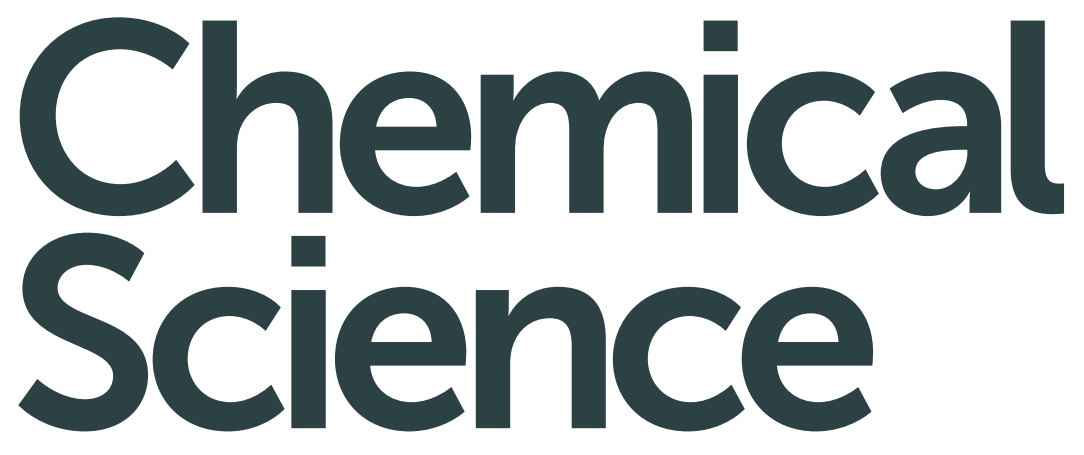

rsc.li/chemical-science

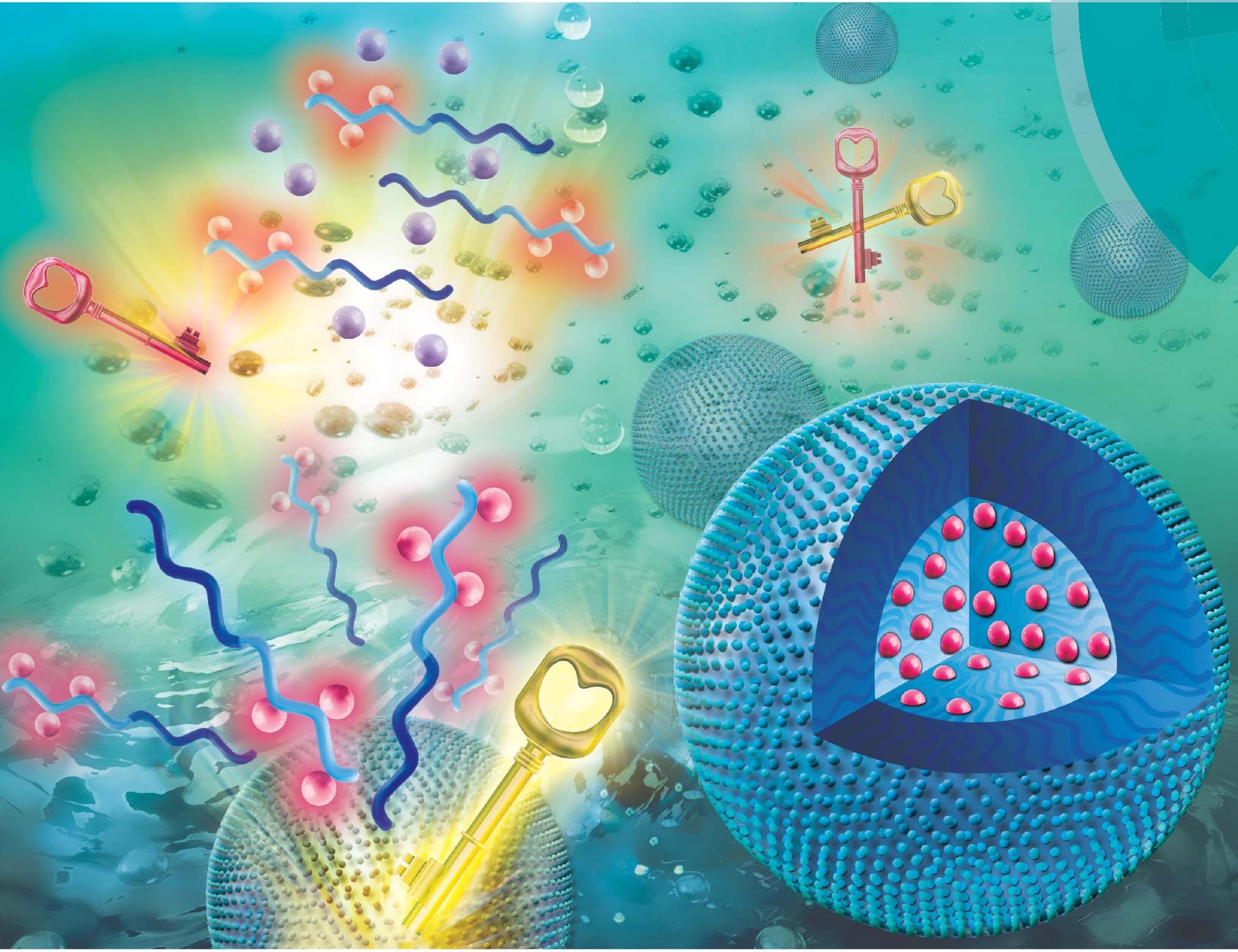

ISSN 2041-6539

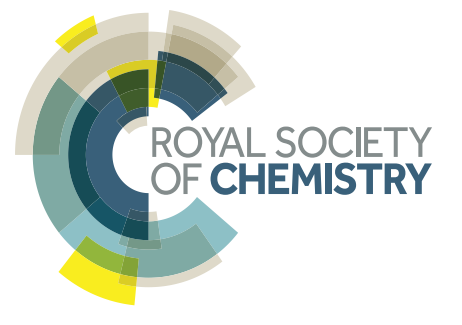


Check for updates

Cite this: Chem. Sci., 2018, 9, 6176

๑ All publication charges for this article have been paid for by the Royal Society of Chemistry

Received 9th May 2018

Accepted 21st June 2018

DOI: $10.1039 / \mathrm{c} 8 \mathrm{sc0} 02079 \mathrm{e}$

rsc.li/chemical-science

\section{A sequence-activated AND logic dual-channel fluorescent probe for tracking programmable drug release $\uparrow$}

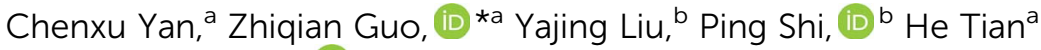 \\ and Wei-Hong Zhu (DD *a
}

\begin{abstract}
The translation of biomarker sensing into programmable diagnostics or therapeutic applications in vivo is greatly challenging, especially for eliminating the 'false positive' signals from OR logic gates. Herein we present a sense-of-logic dual-channel nanoprobe, operating through a sequence-activated AND logic gate by responding ultra-sensitively to $\mathrm{pH}$ changes and being subsequently triggered with biothiol for the controllable release of anti-cancer drugs. Specifically, programmable drug release is conducted in a multistage tumor microenvironment (acidic endocytic organelles followed by abnormal glutathioneoverexpressing cell cytosol), which is synchronous with dual-channel near-infrared (NIR) fluorescence output. This approach represents the merging of sensing and release, including logically enabled molecular design, biomarker sensing, and controllable drug release. Impressively, the sequential AND logic feature within an unprecedented framework provides feedback on the diversity and complexity of biological milieu, along with remarkably enhancing the tumor therapeutic efficiency via its precise targeting ability and programmable drug release.
\end{abstract}

\section{Introduction}

Smart nanoprobes capable of intracellular information processing provide exceptional opportunities for biomarker sensing, molecular imaging, ${ }^{1-10}$ and drug delivery. ${ }^{11-20}$ To date, it is still a tremendous challenge to differentiate concurrent biomarkers, such as dysregulated $\mathrm{pH}$, matrix metalloproteinase activity and aberrant biothiol levels. Current multi-stimulusresponsive probes are predominantly operated by "OR" logic gates in response to each stimulus. ${ }^{21-24}$ However, nonspecific activation in complex biological milieu always leads to "false positive" signals with difficulty in accurate recognition. ${ }^{25,26}$ Inspired by the Boolean logic idea, ${ }^{27-30}$ we envision that the incorporation of sequence-activated fluorescence responses from biomarkers might afford precise behavior in vivo, then produce specific output information for intelligent recognition. A small number of AND logic-based prodrugs have been reported, but they still lack the ability to couple the sequencedependent response to concurrent biomarkers with

${ }^{a}$ Key Laboratory for Advanced Materials and Institute of Fine Chemicals, Shanghai Key Laboratory of Functional Materials Chemistry, School of Chemistry and Molecular Engineering, East China University of Science and Technology, Shanghai, 200237, China.E-mail:whzhu@ecust.edu.cn; guozq@ecust.edu.cn

${ }^{b}$ State Key Laboratory of Bioreactor Engineering, East China University of Science and Technology, Shanghai, 200237, China

$\uparrow$ Electronic supplementary information (ESI) available. See DOI: $10.1039 / \mathrm{c} 8 \mathrm{sc} 02079 \mathrm{e}$ specificity. ${ }^{31,32}$ In diagnostics or therapeutic applications, a sequential AND logic fluorescent nanoprobe that enables the translation of biomarker sensing into a programmable response in vivo has not yet been reported.

Herein we present a proof-of-concept study of a sequenceactivated AND logic dual-channel near-infrared (NIR) fluorescent probe $\mathrm{P}(\mathrm{Cy}-\mathrm{S}-\mathrm{CPT})$, which functions as a programmable sensor and then releases anticancer drugs. To demonstrate the feasibility of our strategy (Fig. 1), two tumor-associated biomarkers, low cellular $\mathrm{pH}$ and overexpressed glutathione (GSH) ${ }^{33-35}$ served as the model stimuli. The smart nanoprobe $\mathrm{P}(\mathrm{Cy}-\mathrm{S}-\mathrm{CPT})$ is composed of two functional components: an ionizable tertiary amine-containing diblock copolymer which renders an ultra-sensitive response to small $\mathrm{pH}$ differences between acidic tumor cells and blood, ${ }^{36-39}$ and a dual-channel NIR fluorescence component Cy-S-CPT for tracking the biothiol-triggered prodrug release in vivo. In Cy-S-CPT, the cyanine (Cy) derivative served as the dual-channel near-infrared fluorophore and the disulfide-bridged anticancer prodrug camptothecin (-S-CPT) served as the activatable unit. As expected, the nanoprobe becomes activatable and detectable with $\mathrm{pH}$ changes, resulting in the subsequent overexpression of GSH coupled with acidic $\mathrm{pH}$, leading to another NIR readout channel (Fig. 1). Specifically, only when the $\mathrm{pH}$ and biothiol stimuli are sequence-dependently triggered is the drug release conducted in a multistage tumor microenvironment (acidic endocytic organelles followed by abnormal GSH-overexpressing cell cytosol), which is synchronous with in vivo dual-channel NIR 

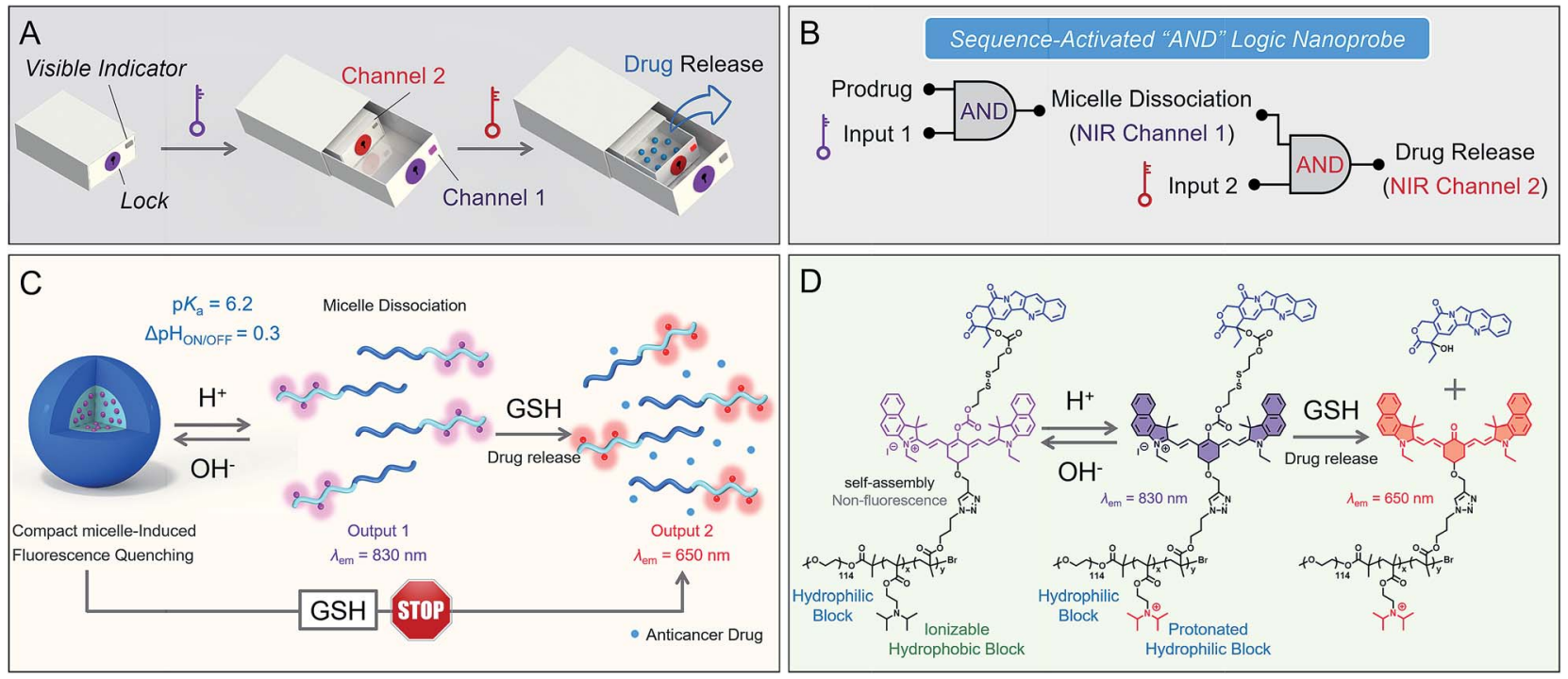

Fig. 1 Sequence-activated AND logic dual-channel NIR fluorescent probe. (A and B) Logic circuit diagram of the sequence-activated AND logic nanoprobe for real-time tracking and programmable drug release via a dual NIR channel. ( $C$ and D) Schematic design of the sequence-activated AND logic dual-channel NIR theranostic nanoprodrug. At $\mathrm{pH}>\mathrm{p} K_{\mathrm{a}}$, the probe self-assembles as a compact micelle, leading to fluorescence quenching at $830 \mathrm{~nm}$. At $\mathrm{pH}<\mathrm{p} K_{\mathrm{a}}$, the probe is in a micelle-dissociated state with a dramatic increase in fluorescence at $830 \mathrm{~nm}$. Subsequently, biothiol-driven dual-channel NIR fluorescence (a remarkable shift from 830 to $650 \mathrm{~nm}$ ) occurs for tracking the programmable responses in synergy with active camptothecin (CPT) release.

fluorescence output. Notably, this strategy would protect prodrugs from destructive environments and undesirable interactions, and would ensure the initiation of designated drug release when needed.

\section{Results and discussion}

\section{Activatable dual-channel NIR fluorescence signals}

Firstly, we focus on whether P(Cy-S-CPT) could sense subtle $\mathrm{pH}$ differences between the acidic endocytic organelles of tumor cells (5.0-6.0) and blood (7.4). Notably, P(Cy-S-CPT) displays a sharp and reversible $\mathrm{pH}$ transition $\left(\mathrm{p} K_{\mathrm{a}}=6.3, \Delta \mathrm{pH}_{\mathrm{ON} / \mathrm{OFF}}=\right.$ 0.3 , Fig. $2 \mathrm{~A}$ and $\mathrm{S} 1$ in the ESI†े). At pH 7.4, P(Cy-S-CPT) forms a compact self-assembled micelle with a spherical morphology, which completely silences the fluorophores ${ }^{40}$ (Fig. 2A and B). Moreover, the aggregation-caused NIR decrease of the monomer Cy-S-CPT confirms the micellization-induced quenching effect (Fig. S2 $\dagger$ ). As expected, when the pH is decreased to 6.0 below the probe's transition, the protonation of the hydrophobic block makes the original compact micelle disassemble, and then leads to a dramatic NIR fluorescence enhancement at $830 \mathrm{~nm}$ (Fig. 2A and D). Thus, P(Cy-S-CPT) could be established as an ultra-sensitive $\mathrm{pH}$-activatable NIR nanoprobe, which remains silent in the blood circulation but performs selective fluorescence activation in acidic tumor microenvironments.

Subsequently, biothiol-driven dual-channel NIR fluorescence (a remarkable shift from 830 to $650 \mathrm{~nm}$ ) was investigated for tracking the programmable responses in synergy with active CPT release. P(Cy-S-CPT) was initially at $\mathrm{pH} 6.0$ (below its transition) in aqueous solution, wherein the micelle disassembled with remarkable NIR emission at $830 \mathrm{~nm}$. Upon the addition of GSH (2 mM), P(Cy-S-CPT) containing disulfide linkages displayed an obvious wavelength-shifting change in both the absorption and emission spectra, ${ }^{41,42}$ along with a color change from green to purple-red. The absorption peak at $730 \mathrm{~nm}$ decreased sharply, and a new band centered at $530 \mathrm{~nm}$ was observed, along with a distinct isosbestic point at $635 \mathrm{~nm}$ (Fig. 2F). Concomitantly, a remarkable hypsochromic shift was also observed in the emission spectra. That is, an NIR fluorescence band at $830 \mathrm{~nm}$ was decreased, accompanied by a sharp increase at $650 \mathrm{~nm}$ (Fig. 2G and H). It took within 16 min to reach the reaction equilibrium at $37^{\circ} \mathrm{C}$. In this case, the dualchannel NIR fluorescence responses at $\mathrm{pH} 6.0$ make it possible to simultaneously track where, when, and how the prodrug is activated and released in vivo (Fig. 1).

\section{Sequence-activated AND logic behaviors}

Unprecedentedly, $\mathrm{P}(\mathrm{Cy}-\mathrm{S}-\mathrm{CPT})$ shows quite different GSH responses at $\mathrm{pH}$ levels above its transition $(\mathrm{pH}>6.3)$. At $\mathrm{pH}$ 7.4, we found that $\mathrm{P}(\mathrm{Cy}-\mathrm{S}-\mathrm{CPT})$ produced neither colorimetric nor fluorescence spectral changes upon the addition of GSH (Fig. $2 \mathrm{C}, \mathrm{K}$ and $\mathrm{L}$ ). This could be ascribed to the preferentially self-assembled compact micelles of $\mathrm{P}(\mathrm{Cy}-\mathrm{S}-\mathrm{CPT})$ at $\mathrm{pH}$ 7.4, which thereby reduced the permeation of GSH into the hydrophobic core. Consequently, the release of active camptothecin (CPT) is effectively hampered. Also, if GSH is absent, P(Cy-SCPT) as a prodrug remains in an intact state regardless of its micelle self-assembly or disassembly (Fig. 2I and J). All TEM images and HPLC analyses provided solid evidence for the aforementioned activation process (Fig. S3 and S4†).

As such, an AND logic gate was built up so that the output active CPT is produced only if $\mathrm{H}^{+}$and GSH are both present as 

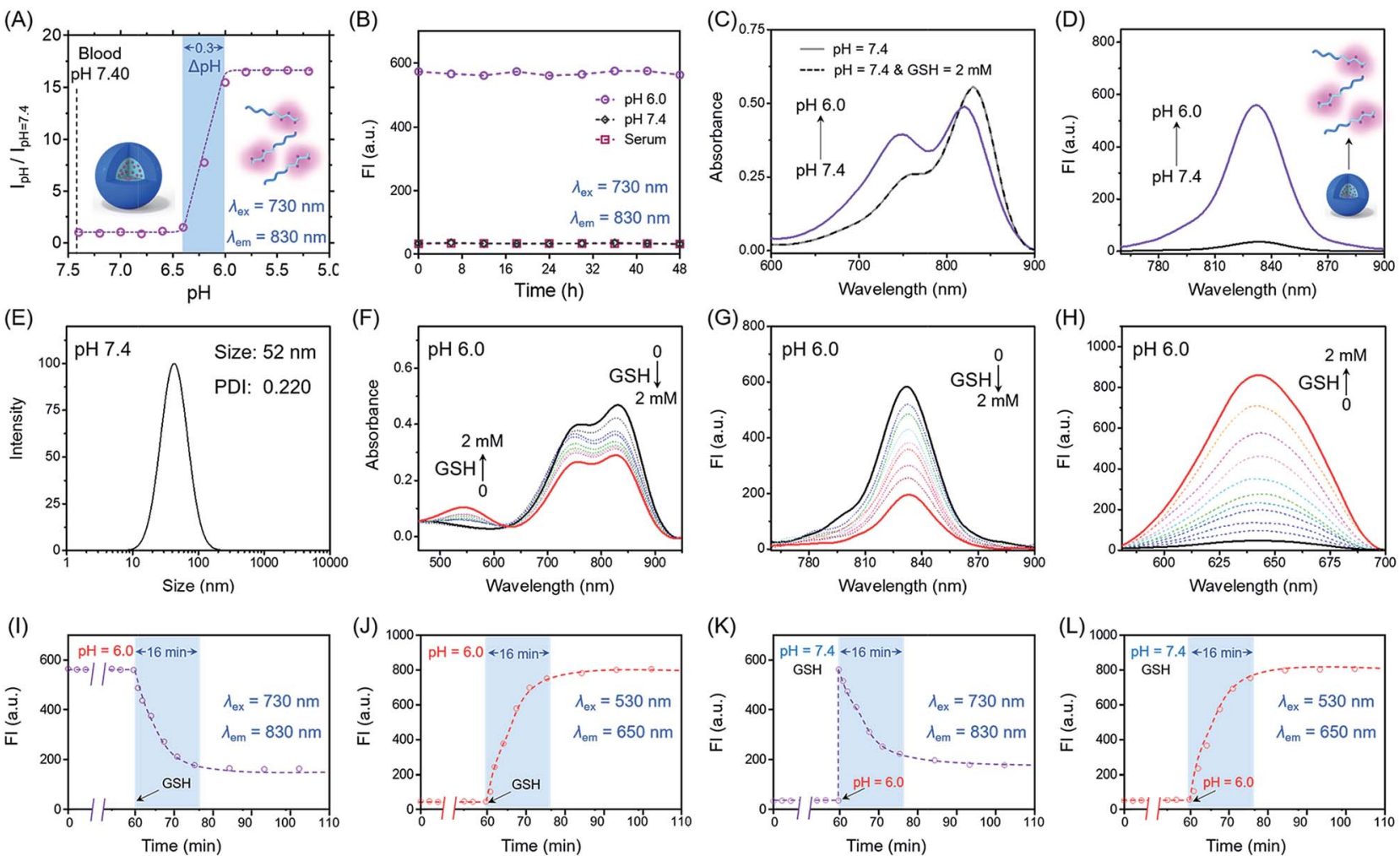

Fig. 2 Self-assembly and dual-channel response of the NIR fluorescent probe. (A) Fluorescence intensity as a function of pH for P(Cy-S-CPT). At a high $\mathrm{pH}$ (for example, 7.4), $\mathrm{P}(\mathrm{Cy}-\mathrm{S}-\mathrm{CPT})$ remains silent. At a $\mathrm{pH}$ below its transition $(\mathrm{pH}=6.3)$, the nanoprobe can be activated as a result of micelle dissociation. For $\mathrm{P}(\mathrm{Cy}-\mathrm{S}-\mathrm{CPT})$, the $\mathrm{pH}$ response is extremely sharp $(\Delta \mathrm{pH}$ ON/OFF $=0.3)$. (B) The $\mathrm{P}(\mathrm{Cy}-\mathrm{S}-\mathrm{CPT})$ nanoprobe remains stable in PBS and human serum over $48 \mathrm{~h}$ at $37^{\circ} \mathrm{C}$. Absorption (C) and emission spectra (D) with $\lambda_{\text {ex }}=730 \mathrm{~nm}$ of $\mathrm{P}(\mathrm{Cy}-\mathrm{S}-\mathrm{CPT})$ in aqueous solution at pH 7.4 (with and without GSH) and 6.0. (E) Size distribution of P(Cy-S-CPT) in aqueous solution at pH 7.4. The absorption spectra (F) and fluorescence emission spectra with $(G) \lambda_{e x}=730 \mathrm{~nm}$ and $(H) \lambda_{e x}=530 \mathrm{~nm}$ of P(Cy-S-CPT) in the presence of GSH (each point was recorded after exposure to $\mathrm{GSH}$ for $15 \mathrm{~min}$ at $37^{\circ} \mathrm{C}$ ) in aqueous solution at $\mathrm{pH} \mathrm{6.0.} \mathrm{(I-L)} \mathrm{Time} \mathrm{dependence} \mathrm{of} \mathrm{fluorescence} \mathrm{intensity} \mathrm{of} \mathrm{P(Cy-S-CPT)} \mathrm{(I} \mathrm{and} \mathrm{J)} \mathrm{in}$ aqueous solution at $\mathrm{pH} 6.0$, with GSH added at $1 \mathrm{~h}$ and $(\mathrm{K}$ and $\mathrm{L})$ in the presence of $\mathrm{GSH}$, adjusting the $\mathrm{pH}$ from 7.4 to 6.0 at $1 \mathrm{~h}$ at 830 and $650 \mathrm{~nm}$.

dual inputs. In particular, a programmable fluorescence response was carried out by the two sequence-dependent inputs (Fig. 1A and B). Specifically, output 1 is fluorescence at $830 \mathrm{~nm}$ (NIR Channel 1) which indicates whether the nanoprobe becomes activated and converts to a secondary form (disassembly); subsequently coupled to the second input of GSH, the disassembled nanoprobe exhibits another readout fluorescence at $650 \mathrm{~nm}$ (NIR Channel 2) as output 2 and the concomitant release of active CPT (Fig. 1B and C). Taken all together, the sequential AND logic gate is successfully constructed, providing a promising strategy for selective sensing and targeted drug release.

To characterize the nanostructure of the self-assembled nanoprobe at $\mathrm{pH}$ 7.4, a solution of $\mathrm{P}(\mathrm{Cy}-\mathrm{S}-\mathrm{CPT})$ was obtained by dialysis. We observed that the assemblies had an average size of $52 \mathrm{~nm}$ measured by dynamic laser scattering (DLS) with a polydispersion index (PDI) of 0.22 (Fig. 2E and S3†). Importantly, these well-defined compact nanoassemblies of $\mathrm{P}(\mathrm{Cy}-\mathrm{S}-$ CPT) maintain their initial good stability over $48 \mathrm{~h}$ in human serum at $37^{\circ} \mathrm{C}$ (Fig. 2B). To gain further insight into their selfassembled behavior, the critical micelle concentration (CMC) of $\mathrm{P}(\mathrm{Cy}-\mathrm{S}-\mathrm{CPT})$ was also measured (Fig. S5†). Notably, the CMC value of $\mathrm{P}(\mathrm{Cy}-\mathrm{S}-\mathrm{CPT})$ is calculated to be only about $0.85 \mu \mathrm{g} \mathrm{mL} \mathrm{m}^{-1}$.
This remarkably low CMC value further confirms the compact and stable nanostructure of $\mathrm{P}(\mathrm{Cy}-\mathrm{S}-\mathrm{CPT})$.

Prior to investigating the programmable responses in living systems, the specificity of sequence-activated $\mathrm{P}(\mathrm{Cy}-\mathrm{S}-\mathrm{CPT})$ for $\mathrm{H}^{+}$ and GSH was evaluated with potential competitive species including amino acids, enzymes, serum markers, metabolic substances and various tissue homogenates (including the heart, liver, spleen, lung and kidney of nude mice). As expected, at a $\mathrm{pH}$ above its transition, $\mathrm{P}(\mathrm{Cy}-\mathrm{S}-\mathrm{CPT})$, which forms a compact micellar structure, did not show any fluorescence response to any of the species (Fig. S6 and S7 7 ). By comparison, only $\mathrm{H}^{+}$elicited a dramatic increase in the fluorescence intensity at $830 \mathrm{~nm}$, suggesting a $\mathrm{pH}$-induced dissociation of the original compact micelle. Furthermore, only upon the addition of $\mathrm{H}^{+}$and then GSH did P(Cy-S-CPT) exhibit an obvious enhancement at another new fluorescence channel of $650 \mathrm{~nm}$, while the propagation of fluorescence at the $830 \mathrm{~nm}$ channel became quenched. Thus, this selective dual-channel fluorescence response promises the preservation of prodrugs from nonspecific activation, while being able to initiate the designated drug release when $\mathrm{H}^{+}$and GSH are sequence-dependently triggered. 


\section{Real-time tracking of each step that leads to drug release in living cells}

As is well known, cancer cells possess acidic endocytic organelles and abnormal GSH-overexpressing cell cytosol. To establish the sequential AND logic behavior in a multistaged tumor environment, A549 cancer cells were incubated with P(Cy-SCPT). As expected, the fluorescent dots at $830 \mathrm{~nm}$ were firstly observed (Fig. 3C), indicating that this nanoprobe was activated and became disassembled inside the endosomes/lysosomes with lower $\mathrm{pH}$ values. Concurrently, there was observed abundant $650 \mathrm{~nm}$ fluorescence in the cytoplasm, indicative of the probe's cleavage by GSH after disassembling. In contrast, after pretreating A549 cells with NEM (GSH inhibitor), the fluorescence of $\mathrm{P}(\mathrm{Cy}-\mathrm{S}-\mathrm{CPT})$ at $830 \mathrm{~nm}$ was still observed while there was no fluorescence at $650 \mathrm{~nm}$ (Fig. 3A). Moreover, after pretreating A549 cells with $\mathrm{NaHCO}_{3}$, the two fluorescence channels of $\mathrm{P}(\mathrm{Cy}-\mathrm{S}-\mathrm{CPT})$ at 830 and $650 \mathrm{~nm}$ were not observed (Fig. 3B). In conjunction with the in vitro results, it can be concluded that we have successfully developed a sequence-activated AND logic

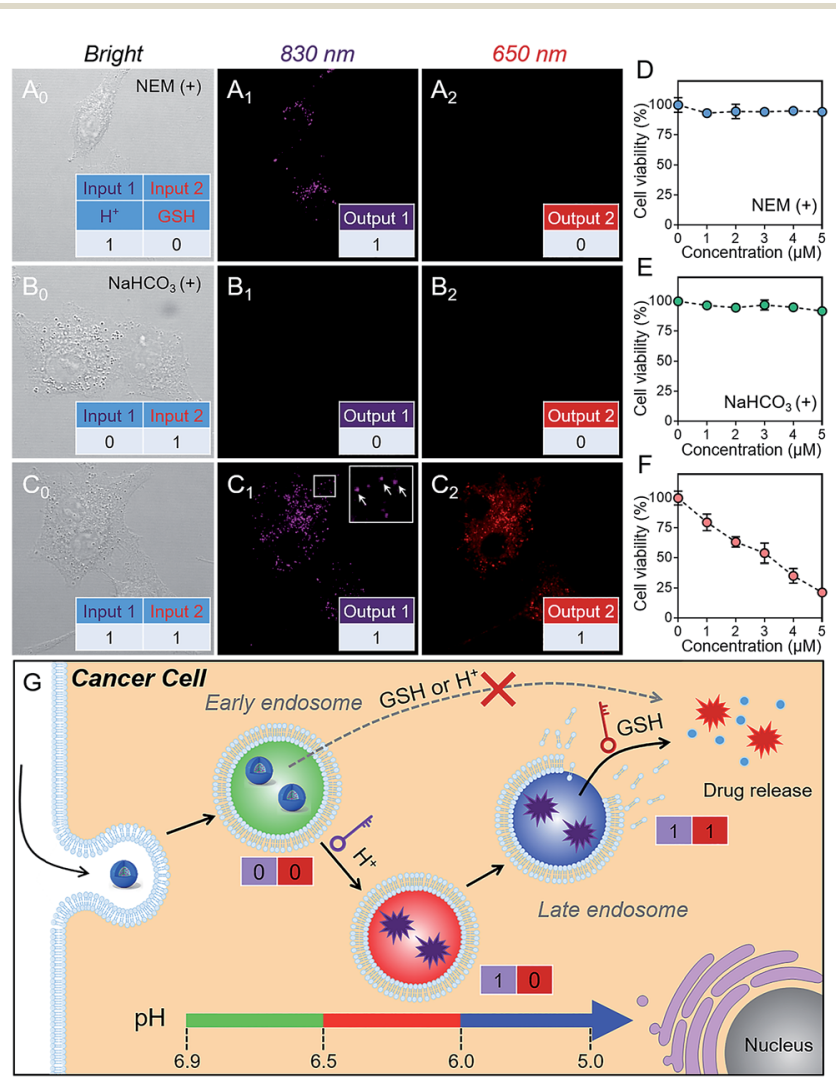

Fig. 3 Dual-channel fluorescence tracking of the programmable drug release in vitro. A549 cancer cells were incubated with NEM \& P(CySCPT) (A), $\mathrm{NaHCO}_{3}$ \& P(Cy-SCPT) (B) and P(Cy-SCPT) (C) at $37{ }^{\circ} \mathrm{C}$ for $1 \mathrm{~h}$. Note: Channel 1: the violet signal $\left(\lambda_{\mathrm{ex}}=730 \mathrm{~nm}, \lambda_{\mathrm{em}}=830 \mathrm{~nm}\right)$ indicates the fluorescence from $\mathrm{H}^{+}$-triggered disassembling; Channel 2 : the red signal $\left(\lambda_{\mathrm{ex}}=530 \mathrm{~nm}, \lambda_{\mathrm{em}}=650 \mathrm{~nm}\right)$ indicates prodrug activation by GSH. A549 cells \& NEM (D), A549 cells \& $\mathrm{NaHCO}_{3}$ (E) and A549 cells (F) were incubated with various concentrations of P(CySCPT) for $24 \mathrm{~h}$. The cell viability was determined using a MTT assay. (G) Schematic design of sequence-activated AND logic dual-channel NIR nanotheranostics. probe for the selective intracellular sensing of a multistage environment (Fig. 3G).

To further validate the biocompatibility, the cytotoxicity of $\mathrm{P}(\mathrm{Cy}-\mathrm{S}-\mathrm{CPT})$ against $\mathrm{A} 549$ cancer cells and normal cell lines was investigated by using a standard MTT assay. As expected, the nanoprobe $\mathrm{P}(\mathrm{Cy}-\mathrm{S}-\mathrm{CPT})$ exhibited a much higher cytotoxicity against cancer cells and no cytotoxicity against normal cells (Fig. S8†). Meanwhile, when A549 cancer cells were preincubated with $\mathrm{NaHCO}_{3}$ or NEM (Fig. 3D-F), P(Cy-S-CPT) exhibited no cytotoxic effects. Taken together, all of these results validated that toxicity results from the $\mathrm{H}^{+}$and $\mathrm{GSH}$ sequential-driven release of active CPT through induced cell apoptosis (Fig. 3G), which is synchronous with dual-channel NIR fluorescence imaging. Clearly, this nanoprobe is selectively intracellularly activated in cancer cells, resulting in programmable drug release and minimizing the side-effects on normal cells.

\section{In vivo dual-channel imaging of the programmable drug release in tumors}

The above promising results inspired us to further explore the feasibility of $\mathrm{P}(\mathrm{Cy}-\mathrm{S}-\mathrm{CPT})$ in living mice. To date, almost all reported drug delivery systems suffer from only one readout channel, which could produce "false positive" information from the programmable behavior of theranostics in vivo. In the prodrug $\mathrm{P}(\mathrm{Cy}-\mathrm{S}-\mathrm{CPT})$, the remarkable shift from the dual-channel response made it perfect to simultaneously track each step of the CPT release. After the intravenous injection of A549 xenograft tumor-bearing mice, in vivo and ex vivo fluorescence bioimaging was recorded. In this way, the in vivo behavior of the small molecule Cy-S-CPT (as a control) was monitored by a dual read-out fluorescence channel (yellow-red represented at $830 \mathrm{~nm}$; rainbow represented at $650 \mathrm{~nm}$ ). As shown in Fig. 4A and B, only a small accumulated amount of Cy-S-CPT was observed at the tumor, but there were strong fluorescence signals from the liver in the dual read-out channel, suggestive of a continuous CPT release that was triggered by nonspecific activation and liver metabolism function towards the small molecular prodrug. In contrast, a specific targeted tumor accumulation of $\mathrm{P}(\mathrm{Cy}-\mathrm{S}-\mathrm{CPT})$ was visualized through both the 830 and $650 \mathrm{~nm}$ fluorescence channels (Fig. 4C and D). This could be attributed to the synergistic effect of the nanoprobe $\mathrm{P}(\mathrm{Cy}-\mathrm{S}-\mathrm{CPT})$ : passive targeting from the EPR effect and activatable targeting (sequence-dependent pH and GSH triggering in a multistage tumor environment).

After $24 \mathrm{~h}$ of intravenous injection, the tumors and major organs of the mice were collected to investigate the biodistribution of Cy-S-CPT and $\mathrm{P}(\mathrm{Cy}-\mathrm{S}-\mathrm{CPT})$, respectively. As shown in Fig. $4 \mathrm{E}$ and $\mathrm{F}$, the dual-channel fluorescence of Cy-SCPT revealed that it is mainly distributed in the liver region, suggestive of a continuous CPT release triggered by liver metabolism function. Comparatively, the ex vivo fluorescence images of the excised tumors with P(Cy-S-CPT) confirmed a high specific accumulation and then activation (Fig. 4G and $\mathrm{H}$ ). Accordingly, there was much weaker fluorescence in the liver and no fluorescence in the other organs (heart, spleen, lung and 

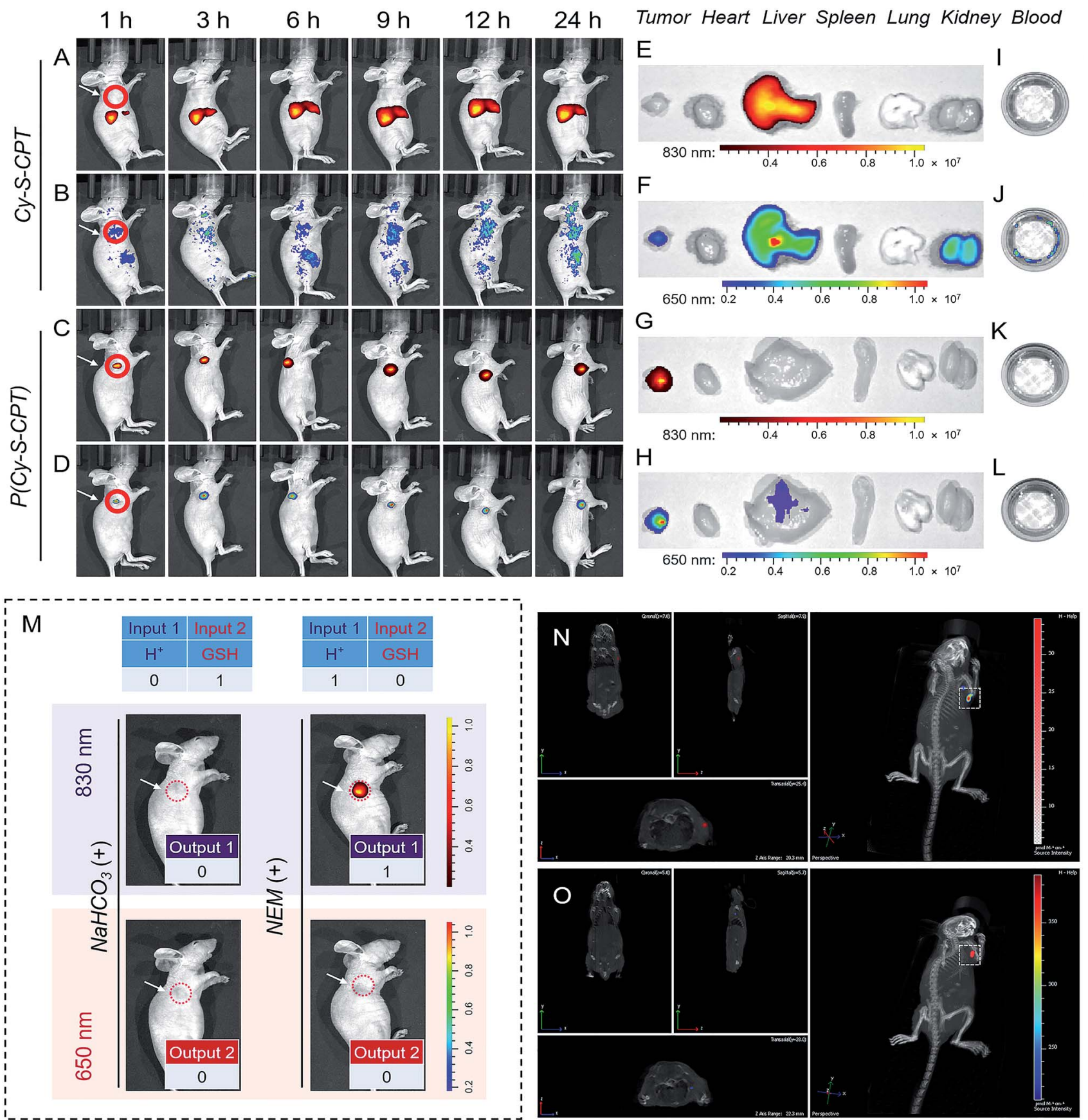

Fig. 4 Dual-channel fluorescence tracking of the programmable drug release in vivo. In vivo dual-channel NIR-fluorescence imaging of A549 xenograft tumor-bearing mice at various times $(1,3,6,9,12$ and $24 \mathrm{~h})$ after the intravenous injection of Cy-S-CPT (A and B) and P(Cy-S-CPT) (C and D) administered at a CPT-equivalent dose of $0.1 \mathrm{mg} \mathrm{kg}^{-1}$. The tumor site is circled in red. Ex vivo NIR-fluorescence imaging of the excised organs (heart, liver, spleen, lung, kidney and tumor) and blood at $24 \mathrm{~h}$ after the intravenous injection of Cy-S-CPT (E, F, I and J) and P(Cy-S-CPT) (G, $\mathrm{H}, \mathrm{K}$ and $\mathrm{L}$ ). (M) $\mathrm{NaHCO}_{3}$ or NEM was intratumorally injected $2 \mathrm{~h}$ before the intravenous injection of P(Cy-S-CPT). Note: fluorescence signals at $830 \mathrm{~nm}$ (yellow-red scale: A, C, E, G, I and K) and $650 \mathrm{~nm}$ (rainbow scale: B, D, F, H, J and L). (N and O) Three-dimensional in vivo imaging after the intravenous injection of P(Cy-S-CPT) for $24 \mathrm{~h}$. Note: NIR fluorescence signals at $830 \mathrm{~nm}(\mathrm{~N}$, white-red scale) and $650 \mathrm{~nm}(\mathrm{O}$, rainbow scale).

kidney). Notably, there was no fluorescence signal in the blood, indicating that $\mathrm{P}(\mathrm{Cy}-\mathrm{S}-\mathrm{CPT})$ undergoes no destruction with a high stability in the circulatory system. Furthermore, for $\mathrm{H}^{+}$ and GSH inhibition control, $\mathrm{NaHCO}_{3}$ or NEM was intratumorally injected $2 \mathrm{~h}$ before $\mathrm{P}(\mathrm{Cy}-\mathrm{S}-\mathrm{CPT})$ administration. As shown in Fig. 4M, sequential AND logic behavior in vivo was achieved in tumor-bearing mice. These programmable dualchannel NIR fluorescence responses are consistent with the cell imaging and MTT assay. All of these results show that $\mathrm{P}(\mathrm{Cy}-$ S-CPT) possesses striking characteristics of having welltargeting properties, and sustained and precisely controllable programmable drug release. 
Real-time three-dimensional imaging is a powerful tool for accurate disease diagnostics, especially for suspicious lesions, with high spatiotemporal precision. The preferable characteristics of $\mathrm{P}(\mathrm{Cy}-\mathrm{S}-\mathrm{CPT})$ with an $830 \mathrm{~nm}$ fluorescence channel (activated by $\mathrm{H}^{+}$) shifting to a $650 \mathrm{~nm}$ fluorescence channel (activated by $\mathrm{H}^{+}$and then GSH) make it suitable for performing real-time three-dimensional bioimaging. After a tail vein injection of $\mathrm{P}(\mathrm{Cy}-\mathrm{S}-\mathrm{CPT})$ into the mice, real-time, high-resolution three-dimensional fluorescence images were obtained with an IVIS Spectrum CT imaging system. As shown in Fig. $4 \mathrm{~N}$ and $\mathrm{O}$ and Video 1 and 2 (in the ESI $\uparrow$ ), $\mathrm{P}(\mathrm{Cy}$-S-CPT) exhibited strong 830 and $650 \mathrm{~nm}$ fluorescence signals in the tumor, thus achieving real-time tracking from different perspectives, showing when the nanoprobe is disassembled and how the active drugs are released in vivo.

To evaluate the efficient tumor accumulation and specific programmable drug release, the in vivo antitumor activity of $\mathrm{P}(\mathrm{Cy}-\mathrm{S}-\mathrm{CPT})$ was systematically studied. Specifically, CPT, Cy-SCPT and P(Cy-S-CPT) were intravenously injected into A549 tumor-bearing mice at a $10 \mathrm{mg} \mathrm{CPT} \mathrm{kg}^{-1}$ dose, and the tumor growth was continuously monitored. As shown in Fig. 5A, in the mice treated with $\mathrm{P}(\mathrm{Cy}-\mathrm{S}-\mathrm{CPT})$, the tumor volume was significantly reduced compared with the control group $(p<0.001)$. More impressively, the tumor in the $\mathrm{P}(\mathrm{Cy}-\mathrm{S}-\mathrm{CPT})$ group was largely eradicated with an inhibition of $93.6 \%$ without there being a noticeable influence on body weight (Fig. 5B, C and D). Obviously, $\mathrm{P}(\mathrm{Cy}-\mathrm{S}-\mathrm{CPT})$ can distinctly reduce the side effects on normal tissues and can promote therapeutic efficiency via accurate recognition and tumor specific programmable drug release.
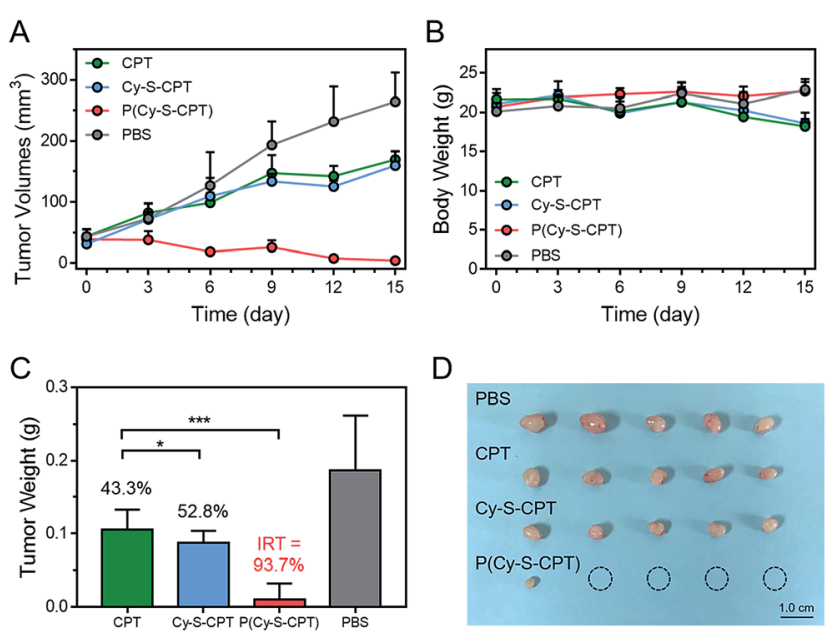

Fig. 5 In vivo antitumor activities of the sequence-activated AND logic nanoprodrug against the A549 tumor xenograft antitumor activities of PBS, CPT, Cy-S-CPT, and P(Cy-S-CPT) administered at a CPT-equivalent dose of $10 \mathrm{mg} \mathrm{kg}^{-1}$ every 3 days against A549 xenograft tumors via intravenous injections. The results are summarized as the tumor volumes of the mice bearing A549 tumors exposed to various treatments (A), body weight changes (B), the tumor weights of each group of the mice at the end of the experiment and the inhibition rates of tumor growth (IRT) $(* p<0.1 ; * * *<0.001)(C)$ and an image of the tumors (D).

\section{Conclusions}

In summary, we developed an unprecedented sequenceactivated AND logic dual-channel NIR fluorescence probe $\mathrm{P}(\mathrm{Cy}-\mathrm{S}-\mathrm{CPT})$ that can sense a multistage tumor environment prior to the release of an active drug. The sense-of-logic dualchannel nanoprobe can stay silent in the blood circulatory system, and is activated synchronously with in vivo dualchannel NIR fluorescence output in response to sequencedependent stimuli such as ultra-small $\mathrm{pH}$ changes $\left(\Delta \mathrm{pH}_{\mathrm{ON} /}\right.$ ofF $=0.3)$ and overexpressed biothiols. The perfect integration of functional sensing and drug release is a breakthrough in the real-time tracking of each step that leads to drug release in vivo, along with three-dimensional bioimaging from the dualchannel NIR fluorescence feedback. Impressively, P(Cy-S-CPT) exhibits excellent multistage tumor targeting ability, as well as a significant enhancement in antitumor activity in vivo, nearly eradicating the tumor. The strategy protects the prodrug from destructive environments and undesirable interactions, while also being able to initiate designated drug release when needed. This sense-of-logic nanoprobe $\mathrm{P}(\mathrm{Cy}-\mathrm{S}-\mathrm{CPT})$ provides a prototype for the development of in vivo intelligent biosensing probes for a precise programmable drug-delivery system.

\section{Conflicts of interest}

There are no conflicts to declare.

\section{Acknowledgements}

This work was supported by NSFC/China (21788102, 21421004, 21636002 and 21622602), the National Key Research and Development Program (2017YFC0906900, 2016YFA02003), Oriental Scholarship, Scientific Committee of Shanghai (14ZR1409700 and 15XD1501400), Shanghai Pujiang Program (13PJD010), the Fok Ying Tong Education Foundation (142014), the Fundamental Research Funds for the Central Universities (WK1013002), and Programme of Introducing Talents of Discipline to Universities (B16017). This study was performed in strict accordance with the NIH guidelines for the care and use of laboratory animals (NIH Publication No. 85-23 Rev. 1985) and was approved by the Institutional Animal Care and Use Committee of the National Tissue Engineering Center (Shanghai, China).

\section{Notes and references}

1 D. Wu, A. C. Sedgwick, T. Gunnlaugsson, E. U. Akkaya, J. Yoon and T. D. James, Chem. Soc. Rev., 2017, 46, 71057123.

2 T. Gunnlaugsson, Nat. Chem., 2016, 8, 6-7.

3 C. Y. Yu, H. Xu, S. Ji, R. T. Kwok, J. W. Lam, X. Li, S. Krishnan, D. Ding and B. Z. Tang, Adv. Mater., 2017, 29, 1606167.

4 X. Li, C. Y. Kim, S. Lee, D. Lee, H. M. Chung, G. Kim, S. H. Heo, C. Kim, K. S. Hong and J. Yoon, J. Am. Chem. Soc., 2017, 139, 10880-10886. 
5 H. S. Jung, J. Han, H. Shi, S. Koo, H. Singh, H. J. Kim, J. L. Sessler, J. Y. Lee, J. H. Kim and J. S. Kim, J. Am. Chem. Soc., 2017, 139, 7595-7602.

6 J. Peng, A. Samanta, X. Zeng, S. Han, L. Wang, D. Su, D. T. Loong, N. Y. Kang, S. J. Park, A. H. All, W. Jiang, L. Yuan, X. Liu and Y. T. Chang, Angew. Chem., Int. Ed., 2017, 56, 4165-4169.

7 Y. Yuan, C. J. Zhang, R. T. K. Kwok, D. Mao, B. Z. Tang and B. Liu, Chem. Sci., 2017, 8, 2723-2728.

8 L. He, X. Yang, K. Xu, X. Kong and W. Lin, Chem. Sci., 2017, 8, 6257-6265.

9 F. Li, J. Lu, X. Kong, T. Hyeon and D. Ling, Adv. Mater., 2017, 29, 1605897.

10 L. K. Truman, S. Comby and T. Gunnlaugsson, Angew. Chem., Int. Ed., 2012, 51, 9624-9627.

11 M. H. Lee, E. J. Kim, H. Lee, H. M. Kim, M. J. Chang, S. Y. Park, K. S. Hong, J. S. Kim and J. L. Sessler, J. Am. Chem. Soc., 2016, 138, 16380-16387.

12 Y. Yuan, R. T. Kwok, B. Z. Tang and B. Liu, J. Am. Chem. Soc., 2014, 136, 2546-2554.

13 M. Ye, Y. Han, J. Tang, Y. Piao, X. Liu, Z. Zhou, J. Gao, J. Rao and Y. Shen, Adv. Mater., 2017, 29, 1702342.

14 L. Cheng, C. Wang, L. Feng, K. Yang and Z. Liu, Chem. Rev., 2014, 114, 10869-10939.

15 X. Wu, Y. J. Tan, H. T. Toh, L. H. Nguyen, S. H. Kho, S. Y. Chew, H. S. Yoon and X. W. Liu, Chem. Sci., 2017, 8, 3980-3988.

16 P. Huang, D. Wang, Y. Su, W. Huang, Y. Zhou, D. Cui, X. Zhu and D. Yan, J. Am. Chem. Soc., 2014, 136, 11748-11756.

17 S. Wang, J. Lin, Z. Wang, Z. Zhou, R. Bai, N. Lu, Y. Liu, X. Fu, O. Jacobson, W. Fan, J. Qu, S. Chen, T. Wang, P. Huang and X. Chen, Adv. Mater., 2017, 29, 1701013.

18 X. Xu, P. E. Saw, W. Tao, Y. Li, X. Ji, S. Bhasin, Y. Liu, D. Ayyash, J. Rasmussen, M. Huo, J. Shi and O. C. Farokhzad, Adv. Mater., 2017, 29, 1700141.

19 J. Li, A. Dirisala, Z. Ge, Y. Wang, W. Yin, W. Ke, K. Toh, J. Xie, Y. Matsumoto, Y. Anraku, K. Osada and K. Kataoka, Angew. Chem., Int. Ed., 2017, 56, 14025-14030.

20 J. Li, Y. Li, Y. Wang, W. Ke, W. Chen, W. Wang and Z. Ge, Nano Lett., 2017, 17, 6983-6990.

21 X. Zhao, C. X. Yang, L. G. Chen and X. P. Yan, Nat. Commun., 2017, 8, 14998.

22 S. Mura, J. Nicolas and P. Couvreur, Nat. Mater., 2013, 12, 991-1003.
23 T. Ma, Y. Hou, J. Zeng, C. Liu, P. Zhang, L. Jing, D. Shangguan and M. Gao, J. Am. Chem. Soc., 2018, 140, 211-218.

24 G. Yang, L. Xu, Y. Chao, J. Xu, X. Sun, Y. Wu, R. Peng and Z. Liu, Nat. Commun., 2017, 8, 902.

25 A. Zakharchenko, N. Guz, A. M. Laradji, E. Katz and S. Minko, Nature Catalysis, 2017, 1, 73-81.

26 Y. Li, S. Sun, L. Fan, S. Hu, Y. Huang, K. Zhang, Z. Nie and S. Yao, Angew. Chem., Int. Ed., 2017, 56, 14888-14892.

27 J. Andréasson and U. Pischel, Chem. Soc. Rev., 2015, 44, 1053-1069.

28 S. Erbas-Cakmak, S. Kolemen, A. C. Sedgwick, T. Gunnlaugsson, T. D. James, J. Yoon and E. U. Akkaya, Chem. Soc. Rev., 2018, 47, 2228-2248.

29 A. P. de Silva, I. M. Dixon, H. Q. N. Gunaratne, T. Gunnlaugsson, P. R. S. Maxwell and T. E. Rice, J. Am. Chem. Soc., 1999, 121, 1393-1394.

30 A. P. de Silva and S. Uchiyama, Nat. Nanotechnol., 2007, 2, 399-410.

31 J. Guo, J. Zhuang, F. Wang, K. R. Raghupathi and S. Thayumanavan, J. Am. Chem. Soc., 2014, 136, 2220-2223.

32 T. Soboleva, H. J. Esquer, A. D. Benninghoff and L. M. Berreau, J. Am. Chem. Soc., 2017, 139, 9435-9438.

33 M. H. Lee, A. Sharma, M. J. Chang, J. Lee, S. Son, J. L. Sessler, C. Kang and J. S. Kim, Chem. Soc. Rev., 2018, 47, 28-52.

34 Z. Liu, X. Zhou, Y. Miao, Y. Hu, N. Kwon, X. Wu and J. Yoon, Angew. Chem., Int. Ed., 2017, 56, 5812-5816.

35 J. Liu, Y. Q. Sun, Y. Huo, H. Zhang, L. Wang, P. Zhang, D. Song, Y. Shi and W. Guo, J. Am. Chem. Soc., 2014, 136, 574-577.

36 J. T. Hou, W. X. Ren, K. Li, J. Seo, A. Sharma, X. Q. Yu and J. S. Kim, Chem. Soc. Rev., 2017, 46, 2076-2090.

37 Y. Wang, K. Zhou, G. Huang, C. Hensley, X. Huang, X. Ma, T. Zhao, B. D. Sumer, R. J. DeBerardinis and J. Gao, Nat. Mater., 2014, 13, 204-212.

38 Z. Wang, M. Luo, C. Mao, Q. Wei, T. Zhao, Y. Li, G. Huang and J. Gao, Angew. Chem., Int. Ed., 2017, 56, 1319-1323.

39 J. Chen, J. Ding, Y. Wang, J. Cheng, S. Ji, X. Zhuang and X. Chen, Adv. Mater., 2017, 29, 1701170.

40 K. Zhou, H. Liu, S. Zhang, X. Huang, Y. Wang, G. Huang, B. D. Sumer and J. Gao, J. Am. Chem. Soc., 2012, 134, 78037811.

41 M. H. Lee, Z. Yang, C. W. Lim, Y. H. Lee, S. Dongbang, C. Kang and J. S. Kim, Chem. Rev., 2013, 113, 5071-5109.

42 M. Z. Ye, X. H. Wang, J. B. Tang, Z. Q. Guo, Y. Q. Shen, H. Tian and W. H. Zhu, Chem. Sci., 2016, 7, 4958-4965. 